\title{
Temperature effects on secondary organic aerosol (SOA) from the dark ozonolysis and photo-oxidation of isoprene (Supporting Information)
}

Christopher H. Clark ${ }^{1,2,3}$, Mary Kacarab ${ }^{1,2}$, Shunsuke Nakao ${ }^{1,2}$, Akua Asa-Awuku ${ }^{1,2}$, Kei Sato $^{4}$, and David R. Cocker, $\mathrm{III}^{1,2^{*}}$

${ }^{1}$ University of California-Riverside, College of Engineering-Center for Environmental Research and Technology (CE-CERT), Riverside, California, USA

${ }^{2}$ University of California-Riverside, Department of Chemical and Environmental Engineering, Riverside, California, USA

${ }^{3}$ Naval Surface Warfare Center, Corona Division, Norco, California, USA

${ }^{4}$ Asian Environment Research Group, National Institute for Environmental Studies, Tsukuba, Ibaraki, Japan

* Corresponding Author, University of CaliforniaRiverside, College of Engineering-Center for Environmental Research and Technology (CE-CERT), 1084 Columbia Ave., Riverside, California, 92507 USA, Phone: (951) 781-5791, E-mail: dcocker@cert.ucr.edu 11 pages, 1 table 
Table S1. PILS-ToF mass spectral ion matches with the corresponding correlations experiments in Table 1 and literature references

\begin{tabular}{|c|c|c|c|}
\hline $\begin{array}{c}\text { PILS-ToF } \\
\text { observed } \\
\text { m/z }\end{array}$ & $\begin{array}{c}\text { TOF-MS ion } \\
\text { formula match } \\
{[\mathrm{M}-\mathrm{H}]^{-}}\end{array}$ & Experiment Observed & References \\
\hline 61.9930 & $\mathrm{NO}_{3}$ & EPA1353A,EPA1559A & 1 \\
\hline 69.0352 & $\mathrm{C}_{4} \mathrm{H}_{5} \mathrm{O}$ & EPA1563A, EPA1556A, EPA1566A & 2 \\
\hline 71.0146 & $\mathrm{C}_{3} \mathrm{H}_{3} \mathrm{O}_{2}$ & EPA1563A, EPA1556A, EPA1566A & 2 \\
\hline 72.9941 & $\mathrm{C}_{2} \mathrm{HO}_{3}$ & EPA1563A & \\
\hline 73.0296 & $\mathrm{C}_{3} \mathrm{H}_{5} \mathrm{O}_{2}$ & $\begin{array}{l}\text { EPA1445A, EPA1483A, EPA1563A, } \\
\text { EPA1556A, EPA1566A }\end{array}$ & 2 \\
\hline 75.0095 & $\mathrm{C}_{2} \mathrm{H}_{3} \mathrm{O}_{3}$ & $\begin{array}{l}\text { EPA1445A, EPA1483A, EPA1563A, } \\
\text { EPA1556A, EPA1566A }\end{array}$ & 2 \\
\hline 79.0045 & $\mathrm{C}_{5} \mathrm{H}_{3} \mathrm{O}$ & EPA1483A & \\
\hline 83.0543 & $\mathrm{C}_{5} \mathrm{H}_{7} \mathrm{O}$ & EPA1483A & \\
\hline 85.0300 & $\mathrm{C}_{4} \mathrm{H}_{5} \mathrm{O}_{2}$ & $\begin{array}{l}\text { EPA1467A, EPA1445A, EPA1483A, } \\
\text { EPA1563A, EPA1556A, EPA1566A }\end{array}$ & 2 \\
\hline 87.0095 & $\mathrm{C}_{3} \mathrm{H}_{3} \mathrm{O}_{3}$ & $\begin{array}{l}\text { EPA1467A, EPA1445A, EPA1483A, } \\
\text { EPA1563A, EPA1556A, EPA1566A }\end{array}$ & 2 \\
\hline 89.0247 & $\mathrm{C}_{3} \mathrm{H}_{5} \mathrm{O}_{3}$ & $\begin{array}{l}\text { EPA1467A, EPA1445A, EPA1483A, } \\
\text { EPA1563A, EPA1556A, EPA1566A }\end{array}$ & 2 \\
\hline 91.0043 & $\mathrm{C}_{2} \mathrm{H}_{3} \mathrm{O}_{4}$ & $\begin{array}{l}\text { EPA1467A, EPA1445A, EPA1563A, } \\
\text { EPA1556A, EPA1566A }\end{array}$ & \\
\hline 95.0152 & $\mathrm{C}_{6} \mathrm{H}_{7} \mathrm{O}$ & EPA1483A & \\
\hline 97.0306 & $\mathrm{C}_{5} \mathrm{H}_{5} \mathrm{O}_{2}$ & $\begin{array}{l}\text { EPA1445A, EPA1483A, EPA1563A, } \\
\text { EPA1556A, EPA1566A }\end{array}$ & 3 \\
\hline 98.0360 & $\mathrm{C}_{4} \mathrm{H}_{5} \mathrm{O}_{2}{ }^{13} \mathrm{C}$ & EPA1483A & 3 \\
\hline 99.0438 & $\mathrm{C}_{5} \mathrm{H}_{7} \mathrm{O}_{2}$ & $\begin{array}{l}\text { EPA1467A, EPA1445A, EPA1483A, } \\
\text { EPA1563A, EPA1556A, EPA1566A }\end{array}$ & 3 \\
\hline 101.0253 & $\mathrm{C}_{4} \mathrm{H}_{5} \mathrm{O}_{3}$ & $\begin{array}{l}\text { EPA1467A, EPA1445A, EPA1483A, } \\
\text { EPA1563A, EPA1556A, EPA1566A }\end{array}$ & 2 \\
\hline 103.0147 & $\mathrm{C}_{3} \mathrm{H}_{3} \mathrm{O}_{4}$ & EPA1556A & 2 \\
\hline 103.0499 & $\mathrm{C}_{4} \mathrm{H}_{7} \mathrm{O}_{3}$ & $\begin{array}{l}\text { EPA1467A, EPA1445A, EPA1483A, } \\
\text { EPA1563A, EPA1556A, EPA1566A }\end{array}$ & 3 \\
\hline 105.0189 & $\mathrm{C}_{3} \mathrm{H}_{5} \mathrm{O}_{4}$ & EPA1467A, EPA1483A & \\
\hline 111.0168 & $\mathrm{C}_{5} \mathrm{H}_{3} \mathrm{O}_{3}$ & EPA1566A & 3 \\
\hline 111.0321 & $\mathrm{C}_{6} \mathrm{H}_{7} \mathrm{O}_{2}$ & EPA1483A & \\
\hline 113.0216 & $\mathrm{C}_{5} \mathrm{H}_{5} \mathrm{O}_{3}$ & $\begin{array}{l}\text { EPA1467A, EPA1445A, EPA1483A, } \\
\text { EPA1563A, EPA1556A, EPA1566A }\end{array}$ & 3 \\
\hline 115.0364 & $\mathrm{C}_{5} \mathrm{H}_{7} \mathrm{O}_{3}$ & $\begin{array}{l}\text { EPA1467A, EPA1445A, EPA1483A, } \\
\text { EPA1563A, EPA1556A, EPA1566A }\end{array}$ & 3 \\
\hline 116.9973 & $\mathrm{C}_{3} \mathrm{HO}_{5}$ & EPA1556A & \\
\hline 117.0229 & $\mathrm{C}_{5} \mathrm{H}_{9} \mathrm{O}_{3}$ & $\begin{array}{l}\text { EPA1353A, EPA1467A, EPA1445A, } \\
\text { EPA1556A }\end{array}$ & 4 \\
\hline 117.0436 & $\mathrm{C}_{4} \mathrm{H}_{6} \mathrm{O}_{4}$ & EPA1483A, EPA1563A, EPA1556A & 2 \\
\hline
\end{tabular}




\begin{tabular}{|c|c|c|c|}
\hline $\begin{array}{l}\text { PILS-ToF } \\
\text { observed } \\
\mathbf{m} / \mathbf{z}\end{array}$ & $\begin{array}{l}\text { TOF-MS ion } \\
\text { formula match } \\
{[\mathrm{M}-\mathrm{H}]^{-}}\end{array}$ & Experiment Observed & References \\
\hline 119.0350 & $\mathrm{C}_{4} \mathrm{H}_{7} \mathrm{O}_{4}$ & $\begin{array}{l}\text { EPA1353A, EPA1483A, EPA1563A, } \\
\text { EPA1556A, EPA1566A, EPA1559A }\end{array}$ & 5 \\
\hline 121.0289 & $\mathrm{C}_{7} \mathrm{H}_{5} \mathrm{O}_{2}$ & $\begin{array}{l}\text { EPA1445A, EPA1483A, EPA1563A, } \\
\text { EPA1556A }\end{array}$ & \\
\hline 123.0371 & $\mathrm{C}_{7} \mathrm{H}_{7} \mathrm{O}_{2}$ & EPA1467A, EPA1483A & \\
\hline 124.9891 & $\mathrm{C}_{5} \mathrm{HO}_{4}$ & EPA1353A, EPA1559A & \\
\hline 125.1282 & $\mathrm{C}_{9} \mathrm{H}_{17}$ & EPA1353A & \\
\hline 125.9869 & $\mathrm{C}_{4} \mathrm{NO}_{4}$ & EPA1353A, EPA1559A & \\
\hline 126.9938 & $\mathrm{C}_{5} \mathrm{H}_{3} \mathrm{O}_{4}$ & $\begin{array}{l}\text { EPA1353A, EPA1445A, EPA1483A, } \\
\text { EPA1559A }\end{array}$ & 3 \\
\hline 127.0365 & $\mathrm{C}_{7} \mathrm{H}_{7} \mathrm{O}_{3}$ & EPA1563A & \\
\hline 127.0488 & $\mathrm{C}_{6} \mathrm{H}_{7} \mathrm{O}_{3}$ & EPA1556A, EPA1566A & \\
\hline 129.0243 & $\mathrm{C}_{5} \mathrm{H}_{5} \mathrm{O}_{4}$ & $\begin{array}{l}\text { EPA1353A, EPA1467A, EPA1445A, } \\
\text { EPA1566A, EPA1559A }\end{array}$ & \\
\hline 129.0569 & $\mathrm{C}_{6} \mathrm{H}_{9} \mathrm{O}_{3}$ & EPA1483A, EPA1563A, EPA1556A & \\
\hline 130.0602 & $\mathrm{C}_{5} \mathrm{H}_{9} \mathrm{O}_{3}{ }^{13} \mathrm{C}$ & EPA1483A, EPA1556A, EPA1566A & \\
\hline 131.0342 & $\mathrm{C}_{5} \mathrm{H}_{7} \mathrm{O}_{4}$ & $\begin{array}{l}\text { EPA1467A, EPA1445A, EPA1483A, } \\
\text { EPA1563A, EPA1556A, EPA1566A }\end{array}$ & 3 \\
\hline 133.0154 & $\mathrm{C}_{5} \mathrm{H}_{9} \mathrm{O}_{4}$ & EPA1467A & \\
\hline 133.0252 & $\mathrm{C}_{3} \mathrm{H}_{5} \mathrm{O}_{5}$ & EPA1483A & 2 \\
\hline 133.0169 & $\mathrm{C}_{4} \mathrm{H}_{5} \mathrm{O}_{5}$ & EPA1563A, EPA1556A & \\
\hline 135.0436 & $\mathrm{C}_{8} \mathrm{H}_{7} \mathrm{O}_{2}$ & EPA1467A, EPA1483A, EPA1556A & \\
\hline 137.0630 & $\mathrm{C}_{8} \mathrm{H}_{9} \mathrm{O}_{2}$ & $\begin{array}{l}\text { EPA1467A, EPA1445A, EPA1483A, } \\
\text { EPA1563A, EPA1556A, EPA1566A }\end{array}$ & \\
\hline 139.0513 & $\mathrm{C}_{7} \mathrm{H}_{7} \mathrm{O}_{3}$ & EPA1483A & \\
\hline 141.0572 & $\mathrm{C}_{7} \mathrm{H}_{9} \mathrm{O}_{3}$ & EPA1483A, EPA1563A, EPA1556A & \\
\hline 143.0392 & $\mathrm{C}_{6} \mathrm{H}_{7} \mathrm{O}_{4}$ & EPA1563A, EPA1566A & \\
\hline 143.0556 & $\mathrm{C}_{10} \mathrm{H}_{7} \mathrm{O}$ & EPA1483A & \\
\hline 143.0683 & $\mathrm{C}_{7} \mathrm{H}_{11} \mathrm{O}_{3}$ & EPA1467A, EPA1445A & \\
\hline 143.0683 & $\mathrm{C}_{7} \mathrm{H}_{11} \mathrm{O}_{3}$ & EPA1467A, EPA1445A & 3 \\
\hline 145.0283 & $\mathrm{C}_{5} \mathrm{H}_{5} \mathrm{O}_{5}$ & EPA1556A, EPA1559A & \\
\hline 145.0394 & $\mathrm{C}_{9} \mathrm{H}_{5} \mathrm{O}_{2}$ & EPA1556A, EPA1566A & \\
\hline 145.0517 & $\mathrm{C}_{6} \mathrm{H}_{9} \mathrm{O}_{4}$ & EPA1483A, EPA1563A & \\
\hline 146.9714 & $\mathrm{C}_{4} \mathrm{H}_{3} \mathrm{O}_{6}$ & EPA1353A & \\
\hline 147.0327 & $\mathrm{C}_{5} \mathrm{H}_{7} \mathrm{O}_{5}$ & $\begin{array}{l}\text { EPA1353A, EPA1467A, EPA1445A, } \\
\text { EPA1483A, EPA1563A, EPA1556A, } \\
\text { EPA1566A, EPA1559A }\end{array}$ & 6 \\
\hline 149.0465 & $\mathrm{C}_{5} \mathrm{H}_{9} \mathrm{O}_{5}$ & $\begin{array}{l}\text { EPA1467A, EPA1483A, EPA1556A, } \\
\text { EPA1566A }\end{array}$ & \\
\hline 151.0349 & $\mathrm{C}_{8} \mathrm{H}_{7} \mathrm{O}_{3}$ & EPA1467A & \\
\hline 151.0608 & $\mathrm{C}_{12} \mathrm{H}_{7}$ & EPA1483A & \\
\hline 151.0712 & $\mathrm{C}_{5} \mathrm{H}_{11} \mathrm{O}_{5}$ & EPA1556A & \\
\hline 153.0462 & $\mathrm{C}_{11} \mathrm{H}_{5} \mathrm{O}$ & EPA1566A & \\
\hline 153.0517 & $\mathrm{C}_{8} \mathrm{H}_{9} \mathrm{O}_{3}$ & EPA1467A, EPA1483A & \\
\hline 155.0416 & $\mathrm{C}_{7} \mathrm{H}_{7} \mathrm{O}_{4}$ & EPA1563A, EPA1556A, EPA1566A & \\
\hline 155.0632 & $\mathrm{C}_{8} \mathrm{H}_{11} \mathrm{O}_{3}$ & EPA1483A & \\
\hline
\end{tabular}




\begin{tabular}{|c|c|c|c|}
\hline $\begin{array}{l}\text { PILS-ToF } \\
\text { observed } \\
\mathbf{m} / \mathbf{z}\end{array}$ & $\begin{array}{l}\text { TOF-MS ion } \\
\text { formula match } \\
{[\mathrm{M}-\mathrm{H}]^{-}}\end{array}$ & Experiment Observed & References \\
\hline 157.0451 & $\mathrm{C}_{7} \mathrm{H}_{9} \mathrm{O}_{4}$ & $\begin{array}{l}\text { EPA1467A, EPA1483A, EPA1563A, } \\
\text { EPA1556A, EPA1566A }\end{array}$ & 2 \\
\hline 159.0402 & $\mathrm{C}_{10} \mathrm{H}_{7} \mathrm{O}_{2}$ & $\begin{array}{l}\text { EPA1467A, EPA1483A, EPA1563A, } \\
\text { EPA1556A, EPA1566A }\end{array}$ & \\
\hline 161.0454 & $\mathrm{C}_{9} \mathrm{H}_{5} \mathrm{O}_{3}$ & EPA1467A, EPA1445A & \\
\hline 161.0500 & $\mathrm{C}_{6} \mathrm{H}_{9} \mathrm{O}_{5}$ & $\begin{array}{l}\text { EPA1483A, EPA1563A, EPA1556A, } \\
\text { EPA1566A }\end{array}$ & \\
\hline 163.0345 & $\mathrm{C}_{9} \mathrm{H}_{7} \mathrm{O}_{3}$ & EPA1467A, EPA1445A & \\
\hline 163.0388 & $\mathrm{C}_{5} \mathrm{H}_{7} \mathrm{O}_{6}$ & EPA1556A & \\
\hline 163.0688 & $\mathrm{C}_{6} \mathrm{H}_{11} \mathrm{O}_{5}$ & EPA1556A & \\
\hline 165.0274 & $\mathrm{C}_{8} \mathrm{H}_{5} \mathrm{O}_{4}$ & EPA1467A & \\
\hline 165.0531 & $\mathrm{C}_{5} \mathrm{H}_{9} \mathrm{O}_{6}$ & EPA1483A, EPA1556A & \\
\hline 167.0658 & $\mathrm{C}_{9} \mathrm{H}_{11} \mathrm{O}_{3}$ & EPA1483A, EPA1556A & \\
\hline 169.0581 & $\mathrm{C}_{8} \mathrm{H}_{9} \mathrm{O}_{6}$ & EPA1563A & \\
\hline 169.0734 & $\mathrm{C}_{9} \mathrm{H}_{13} \mathrm{O}_{3}$ & EPA1483A, EPA1556A & \\
\hline 171.0616 & $\mathrm{C}_{8} \mathrm{H}_{11} \mathrm{O}_{4}$ & $\begin{array}{l}\text { EPA1467A, EPA1445A, EPA1483A, } \\
\text { EPA1563A, EPA1556A, EPA1566A }\end{array}$ & \\
\hline 173.0446 & $\mathrm{C}_{7} \mathrm{H}_{9} \mathrm{O}_{5}$ & $\begin{array}{l}\text { EPA1467A, EPA1483A, EPA1563A, } \\
\text { EPA1556A, EPA1566A }\end{array}$ & \\
\hline 175.0405 & $\mathrm{C}_{10} \mathrm{H}_{7} \mathrm{O}_{3}$ & EPA1467A, EPA1563A, EPA1559A & \\
\hline 175.0608 & $\mathrm{C}_{7} \mathrm{H}_{11} \mathrm{O}_{5}$ & EPA1483A, EPA1556A & \\
\hline 175.9861 & $\mathrm{C}_{4} \mathrm{H}_{2} \mathrm{NO}_{7}$ & EPA1353A & \\
\hline 177.0451 & $\mathrm{C}_{6} \mathrm{H}_{9} \mathrm{O}_{6}$ & EPA1467A & \\
\hline 177.0642 & $\mathrm{C}_{10} \mathrm{H}_{9} \mathrm{O}_{3}$ & EPA1556A & \\
\hline 177.0775 & $\mathrm{C}_{7} \mathrm{H}_{13} \mathrm{O}_{5}$ & EPA1566A & \\
\hline 179.0677 & $\mathrm{C}_{6} \mathrm{H}_{11} \mathrm{O}_{6}$ & EPA1556A & \\
\hline 181.0643 & $\mathrm{C}_{9} \mathrm{H}_{9} \mathrm{O}_{4}$ & EPA1556A & \\
\hline 181.0752 & $\mathrm{C}_{10} \mathrm{H}_{13} \mathrm{O}_{3}$ & EPA1483A, EPA1556A & \\
\hline 183.0817 & $\mathrm{C}_{10} \mathrm{H}_{15} \mathrm{O}_{3}$ & $\begin{array}{l}\text { EPA1445A, EPA1483A, EPA1563A, } \\
\text { EPA1556A }\end{array}$ & \\
\hline 183.00032 & $\mathrm{C}_{7} \mathrm{H}_{3} \mathrm{O}_{6}$ & EPA1559A & \\
\hline 185.0619 & $\mathrm{C}_{12} \mathrm{H}_{9} \mathrm{O}_{2}$ & EPA1467A, EPA1445A, EPA1563A & \\
\hline 185.0805 & $\mathrm{C}_{9} \mathrm{H}_{13} \mathrm{O}_{4}$ & EPA1483A, EPA1556A, EPA1566A & \\
\hline 186.0358 & $\mathrm{C}_{7} \mathrm{H}_{8} \mathrm{NO}_{5}$ & EPA1392A, EPA1382A, EPA1375A & \\
\hline 186.0819 & $\mathrm{C}_{8} \mathrm{H}_{9} \mathrm{O}_{4}{ }^{13} \mathrm{C}$ & EPA1483A & \\
\hline 187.0599 & $\mathrm{C}_{8} \mathrm{H}_{11} \mathrm{O}_{5}$ & $\begin{array}{l}\text { EPA1467A, EPA1445A, EPA1483A, } \\
\text { EPA1563A, EPA1556A, EPA1566A }\end{array}$ & \\
\hline 189.0410 & $\mathrm{C}_{7} \mathrm{H}_{9} \mathrm{O}_{6}$ & $\begin{array}{l}\text { EPA1353A, EPA1467A, EPA1445A, } \\
\text { EPA1483A, EPA1563A, EPA1556A, } \\
\text { EPA1559A }\end{array}$ & 2,7 \\
\hline 191.0319 & $\mathrm{C}_{10} \mathrm{H}_{7} \mathrm{O}_{4}$ & EPA1467A, EPA1483A & \\
\hline 191.0768 & $\mathrm{C}_{7} \mathrm{H}_{11} \mathrm{O}_{6}$ & EPA1556A & \\
\hline 193.0407 & $\mathrm{C}_{6} \mathrm{H}_{9} \mathrm{O}_{7}$ & EPA1467A & \\
\hline 195.0519 & $\mathrm{C}_{6} \mathrm{H}_{11} \mathrm{O}_{7}$ & EPA1467A, EPA1556A, EPA1566A & \\
\hline 197.0811 & $\mathrm{C}_{6} \mathrm{H}_{13} \mathrm{O}_{7}$ & EPA1556A & \\
\hline 197.1012 & $\mathrm{C}_{7} \mathrm{H}_{17} \mathrm{O}_{6}$ & EPA1467A & \\
\hline 197.1090 & $\mathrm{C}_{11} \mathrm{H}_{17} \mathrm{O}_{3}$ & EPA1483A & \\
\hline
\end{tabular}




\begin{tabular}{|c|c|c|c|}
\hline $\begin{array}{l}\text { PILS-ToF } \\
\text { observed } \\
\mathbf{m} / \mathbf{z}\end{array}$ & $\begin{array}{l}\text { TOF-MS ion } \\
\text { formula match } \\
{[\mathrm{M}-\mathrm{H}]^{-}}\end{array}$ & Experiment Observed & References \\
\hline 198.1031 & $\mathrm{C}_{10} \mathrm{H}_{17} \mathrm{O}_{3}{ }^{13} \mathrm{C}$ & EPA1483A & \\
\hline 199.0892 & $\mathrm{C}_{13} \mathrm{H}_{11} \mathrm{O}_{2}$ & $\begin{array}{l}\text { EPA1467A, EPA1445A, EPA1483A, } \\
\text { EPA1563A }\end{array}$ & \\
\hline 199.1024 & $\mathrm{C}_{10} \mathrm{H}_{15} \mathrm{O}_{4}$ & EPA1556A & \\
\hline 199.1630 & $\mathrm{C}_{12} \mathrm{H}_{23} \mathrm{O}_{2}$ & EPA1563A & \\
\hline 200.0987 & $\mathrm{C}_{12} \mathrm{H}_{11} \mathrm{O}_{2}{ }^{13} \mathrm{C}$ & EPA1483A & \\
\hline 201.0699 & $\mathrm{C}_{9} \mathrm{H}_{13} \mathrm{O}_{5}$ & $\begin{array}{l}\text { EPA1467A, EPA1445A, EPA1483A, } \\
\text { EPA1563A, EPA1556A, EPA1566A }\end{array}$ & \\
\hline 203.0570 & $\mathrm{C}_{8} \mathrm{H}_{11} \mathrm{O}_{6}$ & $\begin{array}{l}\text { EPA1467A, EPA1483A, EPA1563A, } \\
\text { EPA1556A, EPA1566A }\end{array}$ & \\
\hline 205.0459 & $\mathrm{C}_{11} \mathrm{H}_{9} \mathrm{O}_{4}$ & EPA1467A, EPA1556A & \\
\hline 205.0659 & $\mathrm{C}_{15} \mathrm{H}_{9} \mathrm{O}$ & EPA1483A & \\
\hline 207.0534 & $\mathrm{C}_{7} \mathrm{H}_{11} \mathrm{O}_{7}$ & EPA1467A & \\
\hline 209.0616 & $\mathrm{C}_{14} \mathrm{H}_{9} \mathrm{O}_{2}$ & EPA1467A, EPA1563A & \\
\hline 211.0596 & $\mathrm{C}_{10} \mathrm{H}_{11} \mathrm{O}_{5}$ & EPA1467A, EPA1483A, EPA1556A & \\
\hline 213.0740 & $\mathrm{C}_{10} \mathrm{H}_{13} \mathrm{O}_{5}$ & EPA1467A, EPA1445A, EPA1556A & \\
\hline 215.0383 & $\mathrm{C}_{7} \mathrm{H}_{3} \mathrm{O}_{4}$ & EPA1559A & \\
\hline 215.0858 & $\mathrm{C}_{10} \mathrm{H}_{15} \mathrm{O}_{5}$ & $\begin{array}{l}\text { EPA1467A, EPA1445A, EPA1483A, } \\
\text { EPA1563A, EPA1556A }\end{array}$ & \\
\hline 217.0782 & $\mathrm{C}_{13} \mathrm{H}_{13} \mathrm{O}_{3}$ & $\begin{array}{l}\text { EPA1467A, EPA1483A, EPA1563A, } \\
\text { EPA1556A }\end{array}$ & \\
\hline 219.0530 & $\mathrm{C}_{8} \mathrm{H}_{11} \mathrm{O}_{7}$ & EPA1467A, EPA1563A & \\
\hline 219.0691 & $\mathrm{C}_{12} \mathrm{H}_{11} \mathrm{O}_{4}$ & EPA1483A, EPA1559A & \\
\hline 219.0977 & $\mathrm{C}_{9} \mathrm{H}_{15} \mathrm{O}_{6}$ & EPA1556A & \\
\hline 221.0690 & $\mathrm{C}_{8} \mathrm{H}_{13} \mathrm{O}_{7}$ & $\begin{array}{l}\text { EPA1353A, EPA1467A, EPA1483A, } \\
\text { EPA1559A }\end{array}$ & 5,6 \\
\hline 223.0523 & $\mathrm{C}_{7} \mathrm{H}_{11} \mathrm{O}_{8}$ & EPA1467A & \\
\hline 225.0637 & $\mathrm{C}_{7} \mathrm{H}_{13} \mathrm{O}_{8}$ & EPA1467A, EPA1556A & \\
\hline 227.0554 & $\mathrm{C}_{10} \mathrm{H}_{11} \mathrm{O}_{6}$ & EPA1467A, EPA1483A, EPA1563A & \\
\hline 227.0914 & $\mathrm{C}_{11} \mathrm{H}_{15} \mathrm{O}_{5}$ & EPA1556A & \\
\hline 229.0701 & $\mathrm{C}_{10} \mathrm{H}_{13} \mathrm{O}_{6}$ & $\begin{array}{l}\text { EPA1353A, EPA1467A, EPA1445A, } \\
\text { EPA1483A, EPA1563A, EPA1556A, } \\
\text { EPA1559A }\end{array}$ & \\
\hline 231.0732 & $\mathrm{C}_{10} \mathrm{H}_{15} \mathrm{O}_{6}$ & EPA1563A & \\
\hline 231.0931 & $\mathrm{C}_{11} \mathrm{H}_{15} \mathrm{O}_{5}$ & EPA1556A & \\
\hline 231.1087 & $\mathrm{C}_{14} \mathrm{H}_{15} \mathrm{O}_{3}$ & EPA1483A & \\
\hline 233.0679 & $\mathrm{C}_{9} \mathrm{H}_{13} \mathrm{O}_{7}$ & $\begin{array}{l}\text { EPA1467A, EPA1483A, EPA1563A, } \\
\text { EPA1559A }\end{array}$ & \\
\hline 233.0999 & $\mathrm{C}_{10} \mathrm{H}_{17} \mathrm{O}_{6}$ & EPA1556A & \\
\hline 235.0041 & $\mathrm{C}_{12} \mathrm{H}_{11} \mathrm{O}_{5}$ & EPA1467A, EPA1483A, EPA1559A & \\
\hline 235.1005 & $\mathrm{C}_{9} \mathrm{H}_{15} \mathrm{O}_{7}$ & EPA1556A & \\
\hline 237.0649 & $\mathrm{C}_{8} \mathrm{H}_{15} \mathrm{O}_{8}$ & EPA1467A, EPA1556A, EPA1559A & \\
\hline 239.0774 & $\mathrm{C}_{8} \mathrm{H}_{15} \mathrm{O}_{8}$ & EPA1467A, EPA1556A & \\
\hline 239.1684 & $\mathrm{C}_{10} \mathrm{H}_{23} \mathrm{O}_{6}$ & EPA1445A & \\
\hline 241.0643 & $\mathrm{C}_{11} \mathrm{H}_{13} \mathrm{O}_{6}$ & EPA1467A, EPA1556A & \\
\hline 241.1234 & $\mathrm{C}_{16} \mathrm{H}_{17} \mathrm{O}_{2}$ & EPA1483A & \\
\hline 243.0546 & $\mathrm{C}_{10} \mathrm{H}_{11} \mathrm{O}_{7}$ & EPA1467A, EPA1559A & \\
\hline 243.0656 & $\mathrm{C}_{14} \mathrm{H}_{11} \mathrm{O}_{8}$ & EPA1563A & \\
\hline 243.0834 & $\mathrm{C}_{7} \mathrm{H}_{15} \mathrm{O}_{7}$ & EPA1483A, EPA1556A, EPA1566A & \\
\hline
\end{tabular}




\begin{tabular}{|c|c|c|c|}
\hline $\begin{array}{l}\text { PILS-ToF } \\
\text { observed } \\
\mathbf{m} / \mathbf{z} \\
\end{array}$ & $\begin{array}{l}\text { TOF-MS ion } \\
\text { formula match } \\
{[\mathrm{M}-\mathrm{H}]^{-}}\end{array}$ & Experiment Observed & References \\
\hline 245.0699 & $\mathrm{C}_{10} \mathrm{H}_{13} \mathrm{O}_{7}$ & EPA1483A, EPA1563A, EPA1556A & 2 \\
\hline 247.0792 & $\mathrm{C}_{10} \mathrm{H}_{15} \mathrm{O}_{7}$ & $\begin{array}{l}\text { EPA1467A, EPA1445A, EPA1483A, } \\
\text { EPA1563A, EPA1556A }\end{array}$ & \\
\hline 249.0623 & $\mathrm{C}_{9} \mathrm{H}_{13} \mathrm{O}_{8}$ & $\begin{array}{l}\text { EPA1353A, EPA1467A, EPA1483A, } \\
\text { EPA1563A, EPA1556A, EPA1559A }\end{array}$ & 5,6 \\
\hline 250.0763 & $\mathrm{C}_{8} \mathrm{H}_{12} \mathrm{NO}_{8}$ & & \\
\hline 251.0791 & $\mathrm{C}_{9} \mathrm{H}_{15} \mathrm{O}_{8}$ & EPA1467A, EPA1556A & \\
\hline 252.0506 & $\mathrm{C}_{7} \mathrm{H}_{10} \mathrm{NO}_{9}$ & EPA1559A & \\
\hline 253.0697 & $\mathrm{C}_{10} \mathrm{H}_{15} \mathrm{O}_{8}$ & EPA1467A & 4 \\
\hline 253.1052 & $\mathrm{C}_{10} \mathrm{H}_{21} \mathrm{O}_{7}$ & EPA1556A & \\
\hline 255.0769 & $\mathrm{C}_{10} \mathrm{H}_{21} \mathrm{O}_{7}$ & EPA1467A & \\
\hline 255.1033 & $\mathrm{C}_{12} \mathrm{H}_{15} \mathrm{O}_{6}$ & EPA1556A & \\
\hline 257.0400 & $\mathrm{C}_{10} \mathrm{H}_{9} \mathrm{O}_{8}$ & EPA1559A & \\
\hline 257.1033 & $\mathrm{C}_{8} \mathrm{H}_{17} \mathrm{O}_{9}$ & EPA1556A & \\
\hline 257.1802 & $\mathrm{C}_{14} \mathrm{H}_{25} \mathrm{O}_{4}$ & EPA1483A & \\
\hline 259.0576 & $\mathrm{C}_{10} \mathrm{H}_{11} \mathrm{O}_{8}$ & EPA1559A & \\
\hline 259.0706 & $\mathrm{C}_{7} \mathrm{H}_{15} \mathrm{O}_{9}$ & EPA1467A, EPA1556A & \\
\hline 259.0945 & $\mathrm{C}_{11} \mathrm{H}_{15} \mathrm{O}_{7}$ & EPA1483A & \\
\hline 260.0286 & $\mathrm{C}_{8} \mathrm{H}_{6} \mathrm{NO}_{9}$ & EPA1559A & \\
\hline 261.0078 & $\mathrm{C}_{5} \mathrm{H}_{9} \mathrm{O}_{12}$ & EPA1563A & \\
\hline 261.0786 & $\mathrm{C}_{14} \mathrm{H}_{13} \mathrm{O}_{5}$ & EPA1467A, EPA1483A & \\
\hline 263.0228 & $\mathrm{C}_{12} \mathrm{H}_{7} \mathrm{O}_{7}$ & EPA1563A & \\
\hline 263.0755 & $\mathrm{C}_{10} \mathrm{H}_{15} \mathrm{O}_{8}$ & $\begin{array}{l}\text { EPA1353A, EPA1467A, EPA1483A, } \\
\text { EPA1556A, EPA1559A }\end{array}$ & 5,6 \\
\hline 264.0893 & $\mathrm{C}_{9} \mathrm{H}_{14} \mathrm{NO}_{8}$ & & \\
\hline 265.0681 & $\mathrm{C}_{13} \mathrm{H}_{13} \mathrm{O}_{6}$ & EPA1467A, EPA1556A & \\
\hline 266.0556 & $\mathrm{C}_{8} \mathrm{H}_{12} \mathrm{NO}_{9}$ & EPA1353A, EPA1559A & 5,6 \\
\hline 267.0596 & $\mathrm{C}_{12} \mathrm{H}_{11} \mathrm{O}_{7}$ & EPA1353A, EPA1467A, EPA1559A & \\
\hline 267.1122 & $\mathrm{C}_{9} \mathrm{H}_{15} \mathrm{O}_{9}$ & EPA1556A & \\
\hline 269.0882 & $\mathrm{C}_{9} \mathrm{H}_{17} \mathrm{O}_{9}$ & EPA1467A, EPA1556A & \\
\hline 271.0110 & $\mathrm{C}_{10} \mathrm{H}_{7} \mathrm{O}_{9}$ & EPA1353A, EPA1559A & \\
\hline 271.0370 & $\mathrm{C}_{11} \mathrm{H}_{11} \mathrm{O}_{8}$ & EPA1563A, EPA1566A & \\
\hline 271.1095 & $\mathrm{C}_{12} \mathrm{H}_{15} \mathrm{O}_{7}$ & EPA1556A & \\
\hline 273.1136 & $\mathrm{C}_{8} \mathrm{H}_{17} \mathrm{O}_{10}$ & EPA1556A & \\
\hline 275.0777 & $\mathrm{C}_{11} \mathrm{H}_{15} \mathrm{O}_{8}$ & EPA1467A, EPA1483A & \\
\hline 277.0952 & $\mathrm{C}_{11} \mathrm{H}_{17} \mathrm{O}_{7}$ & EPA1559A & \\
\hline 277.1113 & $\mathrm{C}_{15} \mathrm{H}_{17} \mathrm{O}_{5}$ & EPA1467A & \\
\hline 279.0727 & $\mathrm{C}_{10} \mathrm{H}_{15} \mathrm{O}_{9}$ & EPA1467A, EPA1483A & \\
\hline 280.0834 & $\mathrm{C}_{9} \mathrm{H}_{14} \mathrm{NO}_{9}$ & EPA1559A & \\
\hline 281.0869 & $\mathrm{C}_{10} \mathrm{H}_{17} \mathrm{O}_{9}$ & EPA1467A, EPA1556A & \\
\hline 282.0953 & $\mathrm{C}_{12} \mathrm{H}_{12} \mathrm{NO}_{7}$ & EPA1559A & \\
\hline 283.0803 & $\mathrm{C}_{13} \mathrm{H}_{15} \mathrm{O}_{7}$ & EPA1467A, EPA1556A & \\
\hline 285.0895 & $\mathrm{C}_{13} \mathrm{H}_{17} \mathrm{O}_{7}$ & EPA1467A, EPA1556A & \\
\hline 286.0551 & $\mathrm{C}_{7} \mathrm{H}_{12} \mathrm{NO}_{11}$ & EPA1382A & \\
\hline 287.1224 & $\mathrm{C}_{12} \mathrm{H}_{15} \mathrm{O}_{8}$ & EPA1483A & \\
\hline 288.0359 & $\mathrm{C}_{10} \mathrm{H}_{10} \mathrm{NO}_{9}$ & EPA1353A, EPA1559A & \\
\hline 289.0130 & $\mathrm{C}_{10} \mathrm{H}_{9} \mathrm{O}_{10}$ & EPA1563A & \\
\hline 289.0542 & $\mathrm{C}_{7} \mathrm{H}_{13} \mathrm{O}_{12}$ & EPA1559A & \\
\hline 289.0847 & $\mathrm{C}_{19} \mathrm{H}_{13} \mathrm{O}_{3}$ & EPA1467A & \\
\hline 290.0527 & $\mathrm{C}_{13} \mathrm{H}_{8} \mathrm{NO}_{7}$ & EPA1559A & \\
\hline 291.0765 & $\mathrm{C}_{11} \mathrm{H}_{15} \mathrm{O}_{9}$ & EPA1353A, EPA1467A, EPA1559A & 7 \\
\hline 293.0801 & $\mathrm{C}_{10} \mathrm{H}_{13} \mathrm{O}_{10}$ & EPA1467A & 2 \\
\hline 293.1069 & $\mathrm{C}_{11} \mathrm{H}_{17} \mathrm{O}_{8}$ & EPA1483A & \\
\hline 296.0649 & $\mathrm{C}_{9} \mathrm{H}_{14} \mathrm{~N}_{1} \mathrm{O}_{10}$ & EPA1353A & 7 \\
\hline
\end{tabular}




\begin{tabular}{|c|c|c|c|}
\hline $\begin{array}{l}297.1227 \\
297.2479 \\
\end{array}$ & $\begin{array}{l}\mathrm{C}_{14} \mathrm{H}_{17} \mathrm{O}_{7} \\
\mathrm{C}_{17} \mathrm{H}_{29} \mathrm{O}_{4} \\
\end{array}$ & $\begin{array}{l}\text { EPA1556A } \\
\text { EPA1445A }\end{array}$ & \\
\hline $\begin{array}{l}\text { PILS-ToF } \\
\text { observed } \\
\mathrm{m} / \mathbf{z} \\
\end{array}$ & 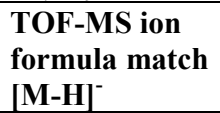 & Experiment Observed & References \\
\hline 298.0901 & $\mathrm{C}_{9} \mathrm{H}_{16} \mathrm{NO}_{10}$ & EPA1559A & \\
\hline 299.1201 & $\mathrm{C}_{10} \mathrm{H}_{29} \mathrm{O}_{10}$ & EPA1556A & \\
\hline 301.0857 & $\mathrm{C}_{12} \mathrm{H}_{13} \mathrm{O}_{9}$ & EPA1556A & \\
\hline 301.1303 & $\mathrm{C}_{14} \mathrm{H}_{21} \mathrm{O}_{7}$ & EPA1483A & \\
\hline 303.1205 & $\mathrm{C}_{13} \mathrm{H}_{19} \mathrm{O}_{8}$ & EPA1483A & \\
\hline 304.0260 & $\mathrm{C}_{10} \mathrm{H}_{10} \mathrm{NO}_{10}$ & EPA1353A, EPA1559A & 7 \\
\hline 305.0867 & $\mathrm{C}_{12} \mathrm{H}_{17} \mathrm{O}_{9}$ & EPA1353A, EPA1467A, EPA1559A & 7 \\
\hline 307.0718 & $\mathrm{C}_{11} \mathrm{H}_{15} \mathrm{O}_{10}$ & EPA1353A, EPA1467A, EPA1559A & 7 \\
\hline 308.1061 & $\mathrm{C}_{10} \mathrm{H}_{14} \mathrm{NO}_{10}$ & EPA1382A & \\
\hline 309.0910 & $\mathrm{C}_{11} \mathrm{H}_{17} \mathrm{O}_{10}$ & EPA1467A & \\
\hline 311.2097 & $\mathrm{C}_{14} \mathrm{H}_{31} \mathrm{O}_{7}$ & EPA1483A, EPA1563A & \\
\hline 312.0603 & $\mathrm{C}_{9} \mathrm{H}_{14} \mathrm{NO}_{11}$ & EPA1353A, EPA1559A & \\
\hline 313.0488 & $\mathrm{C}_{13} \mathrm{H}_{13} \mathrm{O}_{9}$ & EPA1563A, EPA1556A & \\
\hline 315.1410 & $\mathrm{C}_{10} \mathrm{H}_{19} \mathrm{O}_{11}$ & EPA1556A & \\
\hline 315.1372 & $\mathrm{C}_{18} \mathrm{H}_{19} \mathrm{O} 5$ & EPA1483A & \\
\hline 317.0411 & $\mathrm{C}_{12} \mathrm{H}_{13} \mathrm{O}_{10}$ & EPA1563A & \\
\hline 317.1255 & $\mathrm{C}_{14} \mathrm{H}_{17} \mathrm{O}_{10}$ & EPA1483A & \\
\hline 318.0381 & $\mathrm{C}_{9} \mathrm{H}_{14} \mathrm{NO}_{11}$ & EPA1559A & \\
\hline 319.0997 & $\mathrm{C}_{9} \mathrm{H}_{19} \mathrm{O}_{12}$ & EPA1467A, EPA1559A & \\
\hline 321.0861 & $\mathrm{C}_{12} \mathrm{H}_{17} \mathrm{O}_{10}$ & EPA1353A, EPA1467A, EPA1559A & 7 \\
\hline 322.1235 & $\mathrm{C}_{8} \mathrm{H}_{20} \mathrm{NO}_{12}$ & & \\
\hline 323.0976 & $\mathrm{C}_{12} \mathrm{H}_{19} \mathrm{O}_{10}$ & EPA1353A, EPA1467A, EPA1559A & 5,6 \\
\hline 327.0699 & $\mathrm{C}_{9} \mathrm{H}_{15} \mathrm{~N}_{2} \mathrm{O}_{11}$ & EPA1353A, EPA1559A & 5 \\
\hline 328.0931 & $\mathrm{C}_{13} \mathrm{H}_{14} \mathrm{NO}_{9}$ & EPA1559A & \\
\hline 329.1092 & $\mathrm{C}_{14} \mathrm{H}_{17} \mathrm{O}_{9}$ & EPA1556A & \\
\hline 329.10710 & $\mathrm{C}_{10} \mathrm{H}_{17} \mathrm{O}_{12}$ & EPA1563A & \\
\hline 331.1374 & $\mathrm{C}_{11} \mathrm{H}_{23} \mathrm{O}_{11}$ & EPA1483A & \\
\hline 333.0097 & $\mathrm{C}_{11} \mathrm{H}_{9} \mathrm{O}_{12}$ & EPA1563A & \\
\hline 333.0363 & $\mathrm{C}_{13} \mathrm{H}_{17} \mathrm{O}_{10}$ & $\begin{array}{l}\text { EPA1353A, EPA1467A, EPA1483A, } \\
\text { EPA1556A, EPA1559A }\end{array}$ & 7 \\
\hline 335.0973 & $\mathrm{C}_{13} \mathrm{H}_{19} \mathrm{O}_{10}$ & $\begin{array}{l}\text { EPA1353A, EPA1467A, EPA1556A, } \\
\text { EPA1559A }\end{array}$ & 7 \\
\hline 337.0958 & $\mathrm{C}_{12} \mathrm{H}_{17} \mathrm{O}_{11}$ & EPA1467A & \\
\hline 337.1601 & $\mathrm{C}_{17} \mathrm{H}_{21} \mathrm{O}_{7}$ & EPA1556A & \\
\hline 339.1387 & $\mathrm{C}_{12} \mathrm{H}_{19} \mathrm{O}_{11}$ & EPA1353A, EPA1556A, EPA1559A & \\
\hline 349.0812 & $\mathrm{C}_{13} \mathrm{H}_{17} \mathrm{O}_{11}$ & EPA1353A, EPA1467A, EPA1559A & \\
\hline 349.1545 & $\mathrm{C}_{14} \mathrm{H}_{21} \mathrm{O}_{10}$ & EPA1556A & \\
\hline 350.1075 & $\mathrm{C}_{16} \mathrm{H}_{14} \mathrm{NO}_{8}$ & EPA1559A & \\
\hline 351.0956 & $\mathrm{C}_{13} \mathrm{H}_{19} \mathrm{O}_{11}$ & $\begin{array}{l}\text { EPA1353A, EPA1467A, EPA1556A, } \\
\text { EPA1559A }\end{array}$ & \\
\hline 352.0969 & $\mathrm{C}_{12} \mathrm{H}_{18} \mathrm{NO}_{11}$ & EPA1353A, EPA1559A & \\
\hline 353.1631 & $\mathrm{C}_{14} \mathrm{H}_{25} \mathrm{O}_{10}$ & EPA1556A & \\
\hline 354.0724 & $\mathrm{C}_{11} \mathrm{H}_{16} \mathrm{NO}_{12}$ & EPA1353A & \\
\hline 357.0986 & $\mathrm{C}_{11} \mathrm{H}_{17} \mathrm{O}_{13}$ & EPA1559A & \\
\hline 363.0960 & $\mathrm{C}_{14} \mathrm{H}_{19} \mathrm{O}_{11}$ & EPA1353A & \\
\hline 364.1384 & $\mathrm{C}_{16} \mathrm{H}_{14} \mathrm{NO}_{9}$ & EPA1559A & \\
\hline 365.1100 & $\mathrm{C}_{14} \mathrm{H}_{21} \mathrm{O}_{11}$ & EPA1353A, EPA1556A, EPA1559A & \\
\hline 366.1010 & $\mathrm{C}_{13} \mathrm{H}_{20} \mathrm{NO}_{11}$ & EPA1353A, EPA1559A & \\
\hline 367.1114 & $\mathrm{C}_{13} \mathrm{H}_{19} \mathrm{O}_{12}$ & & \\
\hline 367.1747 & $\mathrm{C}_{14} \mathrm{H}_{23} \mathrm{O}_{11}$ & EPA1556A & \\
\hline
\end{tabular}




\begin{tabular}{|c|c|c|c|}
\hline $\begin{array}{l}368.0856 \\
369.0887 \\
375.1139 \\
377.1226 \\
\end{array}$ & $\begin{array}{l}\mathrm{C}_{12} \mathrm{H}_{18} \mathrm{NO}_{12} \\
\mathrm{C}_{16} \mathrm{H}_{17} \mathrm{O}_{10} \\
\mathrm{C}_{15} \mathrm{H}_{19} \mathrm{O}_{11} \\
\mathrm{C}_{11} \mathrm{H}_{21} \mathrm{O}_{14}\end{array}$ & $\begin{array}{l}\text { EPA1353A, EPA1559A } \\
\text { EPA1353A, EPA1559A } \\
\text { EPA1559A } \\
\text { EPA1559A }\end{array}$ & \\
\hline $\begin{array}{l}\text { PILS-ToF } \\
\text { observed } \\
\mathbf{m} / \mathbf{z}\end{array}$ & $\begin{array}{l}\text { TOF-MS ion } \\
\text { formula match } \\
{[\mathrm{M}-\mathrm{H}]^{-}}\end{array}$ & Experiment Observed & References \\
\hline 378.0847 & $\mathrm{C}_{13} \mathrm{H}_{16} \mathrm{NO}_{12}$ & EPA1559A & \\
\hline 379.0950 & $\mathrm{C}_{13} \mathrm{H}_{19} \mathrm{~N}_{2} \mathrm{O}_{11}$ & EPA1353A, EPA1559A & \\
\hline 380.1108 & $\mathrm{C}_{13} \mathrm{H}_{18} \mathrm{NO}_{12}$ & EPA1559A & \\
\hline 382.1126 & $\mathrm{C}_{13} \mathrm{H}_{20} \mathrm{NO}_{12}$ & EPA1559A & \\
\hline 384.1236 & $\mathrm{C}_{13} \mathrm{H}_{22} \mathrm{NO}_{12}$ & EPA1559A & \\
\hline 391.1149 & $\mathrm{C}_{15} \mathrm{H}_{19} \mathrm{O}_{12}$ & EPA1559A & \\
\hline 393.1063 & $\mathrm{C}_{15} \mathrm{H}_{21} \mathrm{O}_{12}$ & EPA1353A, EPA1559A & 7 \\
\hline 394.0930 & $\mathrm{C}_{13} \mathrm{H}_{16} \mathrm{NO}_{13}$ & EPA1559A & \\
\hline 395.1062 & $\mathrm{C}_{11} \mathrm{H}_{23} \mathrm{O}_{15}$ & EPA1353A & \\
\hline 400.1241 & $\mathrm{C}_{13} \mathrm{H}_{22} \mathrm{NO}_{13}$ & EPA1559A & \\
\hline 401.1712 & $\mathrm{C}_{18} \mathrm{H}_{25} \mathrm{O}_{10}$ & EPA1483A, EPA1556A & \\
\hline 406.1056 & $\mathrm{C}_{11} \mathrm{H}_{20} \mathrm{NO}_{15}$ & EPA1559A & \\
\hline 407.1186 & $\mathrm{C}_{16} \mathrm{H}_{23} \mathrm{O}_{12}$ & EPA1353A, EPA1559A & 7 \\
\hline 408.1303 & $\mathrm{C}_{14} \mathrm{H}_{18} \mathrm{NO}_{13}$ & EPA1559A & \\
\hline 409.1024 & $\mathrm{C}_{15} \mathrm{H}_{21} \mathrm{O}_{13}$ & EPA1353A & \\
\hline 410.0975 & $\mathrm{C}_{14} \mathrm{H}_{20} \mathrm{NO}_{13}$ & EPA1353A & \\
\hline 413.1982 & $\mathrm{C}_{16} \mathrm{H}_{29} \mathrm{O}_{12}$ & EPA1445A & \\
\hline 415.1093 & $\mathrm{C}_{14} \mathrm{H}_{23} \mathrm{O}_{16}$ & EPA1559A & \\
\hline 415.1833 & $\mathrm{C}_{18} \mathrm{H}_{25} \mathrm{O}_{10}$ & EPA1483A & \\
\hline 417.1237 & $\mathrm{C}_{24} \mathrm{H}_{17} \mathrm{O}_{7}$ & EPA1559A & \\
\hline 417.1850 & $\mathrm{C}_{17} \mathrm{H}_{21} \mathrm{O}_{12}$ & EPA1483A & \\
\hline 420.1021 & $\mathrm{C}_{15} \mathrm{H}_{18} \mathrm{NO}_{13}$ & EPA1559A & \\
\hline 422.1130 & $\mathrm{C}_{15} \mathrm{H}_{20} \mathrm{NO}_{13}$ & EPA1559A & \\
\hline 423.1150 & $\mathrm{C}_{16} \mathrm{H}_{23} \mathrm{O}_{13}$ & EPA1353A, EPA1559A & \\
\hline 425.1244 & $\mathrm{C}_{16} \mathrm{H}_{25} \mathrm{O}_{13}$ & EPA1353A & \\
\hline 427.2134 & $\mathrm{C}_{17} \mathrm{H}_{31} \mathrm{O}_{12}$ & EPA1445A & \\
\hline 429.1011 & $\mathrm{C}_{13} \mathrm{H}_{21} \mathrm{~N}_{2} \mathrm{O}_{14}$ & EPA1353A, EPA1559A & 5 \\
\hline 429.1697 & $\mathrm{C}_{16} \mathrm{H}_{29} \mathrm{O}_{13}$ & EPA1483A & \\
\hline 430.1284 & $\mathrm{C}_{14} \mathrm{H}_{24} \mathrm{NO}_{14}$ & EPA1559A & \\
\hline 435.1182 & $\mathrm{C}_{17} \mathrm{H}_{23} \mathrm{O}_{13}$ & EPA1353A & \\
\hline 437.1253 & $\mathrm{C}_{12} \mathrm{H}_{25} \mathrm{~N}_{2} \mathrm{O}_{15}$ & EPA1353A & \\
\hline 444.1323 & $\mathrm{C}_{14} \mathrm{H}_{22} \mathrm{NO}_{15}$ & EPA1559A & \\
\hline 445.1340 & $\mathrm{C}_{15} \mathrm{H}_{25} \mathrm{O}_{15}$ & EPA1559A & \\
\hline 447.1638 & $\mathrm{C}_{30} \mathrm{H}_{23} \mathrm{O}_{4}$ & EPA1483A & \\
\hline
\end{tabular}




\begin{tabular}{|c|c|c|c|}
\hline $\begin{array}{l}\text { PILS-ToF } \\
\text { observed } \\
\mathbf{m} / \mathbf{z}\end{array}$ & 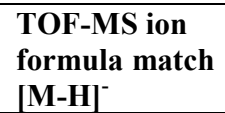 & Experiment Observed & References \\
\hline 451.1133 & $\mathrm{C}_{17} \mathrm{H}_{23} \mathrm{O}_{14}$ & EPA1353A & \\
\hline 453.1250 & $\mathrm{C}_{17} \mathrm{H}_{25} \mathrm{O}_{14}$ & EPA1353A, EPA1559A & \\
\hline 454.1249 & $\mathrm{C}_{16} \mathrm{H}_{24} \mathrm{NO}_{14}$ & EPA1353A & \\
\hline 457.2582 & $\mathrm{C}_{19} \mathrm{H}_{37} \mathrm{O}_{12}$ & EPA1445A & \\
\hline 459.1415 & $\mathrm{C}_{14} \mathrm{H}_{23} \mathrm{~N}_{2} \mathrm{O}_{15}$ & EPA1559A & \\
\hline 461.1603 & $\mathrm{C}_{27} \mathrm{H}_{25} \mathrm{O}_{7}$ & EPA1483A & \\
\hline 465.1252 & $\mathrm{C}_{18} \mathrm{H}_{25} \mathrm{O}_{14}$ & EPA1353A & \\
\hline 467.1386 & $\mathrm{C}_{18} \mathrm{H}_{27} \mathrm{O}_{14}$ & EPA1353A, EPA1559A & 5,6 \\
\hline 468.1215 & $\mathrm{C}_{13} \mathrm{H}_{26} \mathrm{NO}_{17}$ & EPA1353A, EPA1559A & \\
\hline 469.1214 & $\mathrm{C}_{17} \mathrm{H}_{25} \mathrm{O}_{15}$ & EPA1353A & \\
\hline 470.1146 & $\mathrm{C}_{16} \mathrm{H}_{24} \mathrm{NO}_{15}$ & EPA1353A, EPA1559A & 5,6 \\
\hline 471.1178 & $\mathrm{C}_{20} \mathrm{H}_{23} \mathrm{O}_{13}$ & EPA1353A & \\
\hline 471.3020 & $\mathrm{C}_{20} \mathrm{H}_{39} \mathrm{O}_{12}$ & EPA1445A & \\
\hline 472.1185 & $\mathrm{C}_{12} \mathrm{H}_{26} \mathrm{NO}_{18}$ & EPA1353A & \\
\hline 481.1252 & $\mathrm{C}_{18} \mathrm{H}_{25} \mathrm{O}_{15}$ & EPA1353A & \\
\hline 483.1321 & $\mathrm{C}_{13} \mathrm{H}_{27} \mathrm{~N}_{2} \mathrm{O}_{17}$ & EPA1353A & \\
\hline 484.1449 & $\mathrm{C}_{17} \mathrm{H}_{26} \mathrm{NO}_{15}$ & EPA1559A & \\
\hline 495.1360 & $\mathrm{C}_{19} \mathrm{H}_{27} \mathrm{O}_{15}$ & EPA1353A & \\
\hline 496.1335 & $\mathrm{C}_{18} \mathrm{H}_{26} \mathrm{NO}_{15}$ & EPA1353A & \\
\hline 498.1155 & $\mathrm{C}_{17} \mathrm{H}_{24} \mathrm{NO}_{16}$ & EPA1353A & \\
\hline 500.1232 & $\mathrm{C}_{17} \mathrm{H}_{26} \mathrm{NO}_{16}$ & EPA1353A & \\
\hline 509.1464 & $\mathrm{C}_{15} \mathrm{H}_{29} \mathrm{~N}_{2} \mathrm{O}_{17}$ & EPA1353A & \\
\hline 511.1320 & $\mathrm{C}_{19} \mathrm{H}_{27} \mathrm{O}_{16}$ & EPA1353A & \\
\hline 512.1274 & $\mathrm{C}_{18} \mathrm{H}_{26} \mathrm{NO}_{16}$ & EPA1353A & 1 \\
\hline 513.1380 & $\mathrm{C}_{14} \mathrm{H}_{29} \mathrm{~N}_{2} \mathrm{O}_{18}$ & EPA1353A & \\
\hline 525.1438 & $\mathrm{C}_{20} \mathrm{H}_{29} \mathrm{O}_{16}$ & EPA1353A & \\
\hline 527.1491 & $\mathrm{C}_{20} \mathrm{H}_{31} \mathrm{O}_{16}$ & EPA1353A & 5,6 \\
\hline 531.1283 & $\mathrm{C}_{17} \mathrm{H}_{27} \mathrm{~N}_{2} \mathrm{O}_{17}$ & EPA1353A, EPA1559A & 5 \\
\hline 539.1519 & $\mathrm{C}_{17} \mathrm{H}_{31} \mathrm{O}_{19}$ & EPA1353A & \\
\hline 553.1430 & $\mathrm{C}_{21} \mathrm{H}_{29} \mathrm{O}_{17}$ & EPA1353A & \\
\hline 555.1539 & $\mathrm{C}_{21} \mathrm{H}_{31} \mathrm{O}_{17}$ & EPA1353A & 5,6 \\
\hline 556.1522 & $\mathrm{C}_{20} \mathrm{H}_{30} \mathrm{NO}_{17}$ & EPA1353A & \\
\hline $557.0560^{*}$ & $\begin{array}{l}\mathrm{C}_{10} \mathrm{H}_{16} \mathrm{~N}_{5} \mathrm{O}_{18} \\
+\mathrm{NO}_{3}\end{array}$ & & 1 \\
\hline 567.1535 & $\mathrm{C}_{22} \mathrm{H}_{31} \mathrm{O}_{17}$ & EPA1353A & \\
\hline 569.1668 & $\mathrm{C}_{22} \mathrm{H}_{33} \mathrm{O}_{17}$ & EPA1353A & \\
\hline 570.1480 & $\mathrm{C}_{17} \mathrm{H}_{32} \mathrm{NO}_{20}$ & EPA1353A & \\
\hline 572.1445 & $\mathrm{C}_{20} \mathrm{H}_{30} \mathrm{NO}_{18}$ & EPA1353A & 5,6 \\
\hline 573.1476 & $\mathrm{C}_{24} \mathrm{H}_{29} \mathrm{O}_{16}$ & EPA1353A & \\
\hline 583.1538 & $\mathrm{C}_{22} \mathrm{H}_{31} \mathrm{O}_{18}$ & EPA1353A & \\
\hline
\end{tabular}




\begin{tabular}{llll}
\hline $\begin{array}{l}\text { PILS-ToF } \\
\text { observed } \\
\text { m/z }\end{array}$ & $\begin{array}{l}\text { TOF-MS ion } \\
\text { formula match } \\
{[\mathbf{M}-\mathrm{H}]^{-}}\end{array}$ & Experiment Observed & References \\
\hline 597.1638 & $\mathrm{C}_{23} \mathrm{H}_{33} \mathrm{O}_{18}$ & EPA1353A & \\
613.1606 & $\mathrm{C}_{23} \mathrm{H}_{33} \mathrm{O}_{19}$ & EPA1353A & \\
614.1557 & $\mathrm{C}_{22} \mathrm{H}_{32} \mathrm{NO}_{19}$ & EPA1353A & \\
627.1725 & $\mathrm{C}_{24} \mathrm{H}_{35} \mathrm{O}_{19}$ & EPA1353A & 5,6 \\
657.1823 & $\mathrm{C}_{25} \mathrm{H}_{37} \mathrm{O}_{20}$ & EPA1353A & 5,6 \\
671.1933 & $\mathrm{C}_{26} \mathrm{H}_{39} \mathrm{O}_{20}$ & EPA1353A & \\
672.1750 & $\mathrm{C}_{17} \mathrm{H}_{38} \mathrm{NO}_{26}$ & EPA1353A & 5,6 \\
674.1723 & $\mathrm{C}_{24} \mathrm{H}_{36} \mathrm{NO}_{21}$ & EPA1353A & 5,6 \\
675.1766 & $\mathrm{C}_{28} \mathrm{H}_{35} \mathrm{O}_{19}$ & EPA1353A & 5,6 \\
759.2090 & $\mathrm{C}_{29} \mathrm{H}_{43} \mathrm{O}_{23}$ & EPA1353A & 5,6 \\
776.2022 & $\mathrm{C}_{28} \mathrm{H}_{42} \mathrm{NO}_{24}$ & EPA1353A & \\
878.2288 & $\mathrm{C}_{32} \mathrm{H}_{48} \mathrm{NO}_{27}$ & EPA1353A &
\end{tabular}


1. Ng, N. L.; Kwan, A. J.; Surratt, J. D.; Chan, A. W. H.; Chhabra, P. S.; Sorooshian, A.; Pye, H. O. T.; Crounse, J. D.; Wennberg, P. O.; Flagan, R. C.; Seinfeld, J. H., Secondary organic aerosol (SOA) formation from reaction of isoprene with nitrate radicals (NO3). Atmos. Chem. Phys. 2008, 8, (14), $4117-$ 4140 .

2. Nguyen, T. B.; Bateman, A. P.; Bones, D. L.; Nizkorodov, S. A.; Laskin, J.; Laskin, A., Highresolution mass spectrometry analysis of secondary organic aerosol generated by ozonolysis of isoprene. Atmospheric Environment 2010, 44, 1032-1042.

3. $\quad$ Fang, W.; Gong, L.; Zhang, Q.; Cao, M.; Li, Y.; Sheng, L., Measurements of Secondary Organic Aerosol Formed from OH-initiated Photo-oxidation of Isoprene Using Online Photoionization Aerosol Mass Spectrometry. Environmental Science \& Technology 2012, 46, (7), 3898-3904.

4. Lin, Y.-H.; Zhang, Z.; Docherty, K. S.; Zhang, H.; Budisulistiorini, S. H.; Rubitschun, C. L.; Shaw, S. L.; Knipping, E. M.; Edgerton, E. S.; Kleindienst, T. E.; Gold, A.; Surratt, J. D., Isoprene Epoxydiols as Precursors to Secondary Organic Aerosol Formation: Acid-Catalyzed Reactive Uptake Studies with Authentic Compounds. Environmental Science \& Technology 2012, 46, (1), 250-258. 5. Sato, K.; Nakao, S.; Clark, C. H.; Qi, L.; Cocker Iii, D. R., Secondary organic aerosol formation from the photooxidation of isoprene, 1,3-butadiene, and 2,3-dimethyl-1,3-butadiene under high NOx conditions. Atmos. Chem. Phys. 2011, 11, (14), 7301-7317.

6. $\quad$ Surratt, J. D.; Chan, A. W. H.; Eddingsaas, N. C.; Chan, M.; Loza, C. L.; Kwan, A. J.; Hersey, S. P.; Flagan, R. C.; Wennberg, P. O.; Seinfeld, J. H., Reactive intermediates revealed in secondary organic aerosol formation from isoprene. Proceedings of the National Academy of Sciences 2010, 107, (15), 66406645.

7. $\quad$ Nguyen, T. B.; Laskin, J.; Laskin, A.; Nizkorodov, S. A., Nitrogen-containing Organic Compounds and Oligomers in Secondary Organic Aerosol Formed by Photooxidation of Isoprene. Environ. Sci. Technol. 2011, 45, (16), 6908-6918. 